\title{
Fatores que podem influenciar na promoção da saúde do caminhoneiro: uma revisão
}

\section{integrativa}

\author{
Factors that can influence the health promotion of the truck: an integrative review \\ Factores que pueden influir en la promoción de salud del camión: una revisión integrativa
}

Recebido: 22/10/2021 | Revisado: 30/10/2021 | Aceito: 06/11/2021 | Publicado: 10/11/2021

\author{
Lucileide Alves da Silva \\ ORCID: https://orcid.org/0000-0002-3784-259X \\ Centro Universitário Santo Agostinho, Brasil \\ E-mail: leidealves1221@gmail.com \\ Francisco Adalberto do Nascimento Paz \\ ORCID: https://orcid.org/0000-0001-6697-1705 \\ Centro Universitários Santo Agostinho, Brasil \\ E-mail: pazadalberto19@hotmail.com
}

\begin{abstract}
Resumo
Os caminhoneiros fazem parte de uma grande equipe que movimenta a economia do mundo. A má alimentação, a falta de exercícios físicos, as longas jornadas de trabalho sem obedecer ao repouso recomendado e a falta de proteção nas suas relações sexuais com os profissionais do sexo são um grande potencializador para adquirir alguma doença. $\mathrm{O}$ objetivo desse estudo buscou analisar a produção científica acerca fatores que podem influenciar na promoção da saúde do caminhoneiro, o risco de doenças cardiovasculares, nos motoristas de caminhão, conhecer os fatores ocasionados por excesso de longas jornadas de trabalho, alcoolismos e DSTs. O presente estudo trata-se de uma revisão de literatura com pesquisa nas bases de bibliotecas virtuais aplicando os Descritores em Ciências da Saúde (DeCS), a dados coletados foi coletado registrados em formulário com posterior analise por uma revisora da pesquisa. Resultados: foram identificados 1.776 estudos, 937 na MEDLINE, 349 na PUBMED e 490 na SCIELO. Após a análise pela pesquisadora, 61 estudos foram selecionados para leitura. Desses estudos, 50 foram excluídos após a leitura, sendo selecionados 11 estudos validos para a composição da revisão. No Brasil estudos identificaram inúmeros problemas para a saúde do caminhoneiro. As longas jornadas de trabalho com poucas interrupções fazem que o esse profissional acabe por negligenciar a sua saúde. Além disso o desenvolvimento de certos hábitos contribui como fatores de risco para predisposição de várias doenças. Oferecer compreensão da complexidade acerca dos riscos e a exposição aos fatores que influenciam na saúde do caminhoneiro é de grande importância.
\end{abstract}

Palavras-chave: Promoção de saúde; Qualidade de vida; Autocuidado; Transporte.

\begin{abstract}
Truck drivers are part of a great team that moves the world's economy. Poor diet, lack of physical exercise, long working hours without complying with the recommended rest and lack of protection in their sexual relations with sex workers are a great potential for acquiring a disease. The aim of this study was to analyze the scientific production about factors that can influence the health promotion of truck drivers, the risk of cardiovascular disease in truck drivers, to know the factors caused by excessive long work hours, alcoholism and STDs. The present study is a literature review with research in virtual library databases applying the Health Sciences Descriptors (DeCS), the collected data was collected and registered in a form with subsequent analysis by a research reviewer. Results: 1,776 studies were identified, 937 in MEDLINE, 349 in PUBMED and 490 in SCIELO. After analysis by the researcher, 61 studies were selected for reading. Of these studies, 50 were excluded after reading, and 11 valid studies were selected for the composition of the review. In Brazil, studies have identified numerous health problems for truck drivers. Long working hours with few interruptions make this professional end up neglecting his health. Furthermore, the development of certain habits contributes as risk factors for predisposition to various diseases. Offering an understanding of the complexity of risks and exposure to factors that influence the health of truck drivers is of great importance.
\end{abstract}

Keywords: Health Policy; Quality of life; Self-Care; Transportation.

\section{Resumen}

Los camioneros son parte de un gran equipo que mueve la economía mundial. La mala alimentación, la falta de ejercicio físico, las largas jornadas laborales sin cumplir con el descanso recomendado y la falta de protección en las relaciones sexuales con las trabajadoras sexuales son un gran potencial para adquirir una enfermedad. El objetivo de este estudio fue analizar la producción científica sobre los factores que pueden influir en la promoción de la salud de los camioneros, el riesgo de enfermedad cardiovascular en los camioneros, conocer los factores provocados por la excesiva jornada laboral, el alcoholismo y las ETS. El presente estudio es una revisión de la literatura con investigación en bases de datos 
de bibliotecas virtuales aplicando los Descriptores de Ciencias de la Salud (DeCS), los datos recolectados fueron recolectados y registrados en un formulario con posterior análisis por parte de un revisor de la investigación. Resultados: Se identificaron 1.776 estudios, 937 en MEDLINE, 349 en PUBMED y 490 en SCIELO. Después del análisis por parte del investigador, se seleccionaron 61 estudios para lectura. De estos estudios, 50 se excluyeron después de la lectura y se seleccionaron 11 estudios válidos para la composición de la revisión. En Brasil, los estudios han identificado numerosos problemas de salud para los conductores de camiones. Las largas jornadas laborales con pocas interrupciones hacen que este profesional acabe descuidando su salud. Además, el desarrollo de ciertos hábitos contribuye como factor de riesgo de predisposición a diversas enfermedades. Ofrecer una comprensión de la complejidad de los riesgos y la exposición a los factores que influyen en la salud de los conductores de camiones es de gran importancia.

Palabras clave: Política de Salud; Calidad de vida; Autocuidado; Transportes.

\section{Introdução}

Os caminhoneiros fazem parte de uma grande equipe que movimenta a economia do mundo. Da matéria prima até o produto final, praticamente tudo passa por algum caminhão. Nesse entremeio, vale destacar que o desenvolvimento e agravamento das morbidades em caminhoneiros estão documentados em estudos no âmbito teórico-técnico nacional, tendo se relacionado diretamente às suas jornadas extensas de trabalho, exigências no cumprimento dos prazos de entrega, ausência de pausas para descanso, horário irregular de trabalho, trabalho no turno noturno, hábitos de alimentação não saudáveis (Fogaça et al., 2012).

Por conta desses hábitos, alguns motoristas de caminhão apresentam diversos problemas, que por ventura estão relacionados às condições de saúde, trabalho e estilo de vida, além de grande vulnerabilidade, pelo uso das drogas e por possuírem diversas parceiras sexuais diariamente. Tais complicações decorrem das condições de trabalho no qual eles se encontram e do estilo de vida que levam, por se alimentarem de alimentos com baixo índice nutritivo e não praticarem suas refeições corretamente. (Ramos et al., 2018 Estudos realizados mostram que a hipertensão arterial sistêmica (HAS) e a diabetes mellitus (DM) são as principais morbidades que esses profissionais adquirem, ocasionados pelo seu mal hábito (Rodrigues et al., 2018).

Em decorrência das longas e intermináveis horas de viagens, muitas vezes com os prazos abusivos de entregas cargueiras, A grande maioria dos profissionais acabam por ter que se distanciar de sua família pelas condições trabalhistas ou seja; viajar mais no menor tempo possível E sem esse amparo familiar acabam sofrendo de diversas doenças psicológicas como estresse, ansiedade; depressão, solidão, violência no transito e entre outras. Ao se sentirem assim, acabam recorrendo a alcoolismo e as drogas. O uso principal do uso dessas substancias servem para compensar o cansaço e as várias horas de sono perdido. A utilização de certas drogas, fazem que o caminhoneiro fique acordado, pois é um exercício que requer a máxima atenção (Antoniassi Junior et al., 2016).

O caminhoneiro sofre com o ritmo intenso de sua jornada de trabalho diária que lhe é imposta, e esta circunstância propicia maior desgaste físico-mental e emocional-afetivo que afetam sua saúde, causando inúmeros distúrbios para o organismo, consequentemente afetando sua qualidade de vida. O trabalho noturno pode levar a consideráveis efeitos na saúde do trabalhador, com um rápido desgaste individual, devido aos distúrbios provocados nos ritmos biológicos (Silva, Rocha \& Lima, 2019).

Francisco et al. (2018) relata que a obesidade se destaca como um outro agravamento patológico de problemas cardiovasculares e a hipertensão arterial é seis vezes maior em obesos do que em não obesos. Para cada aumento de $10 \%$ no peso corporal, há aumento na incidência de doença coronariana em aproximadamente 20\% (Notto et al., 2017).

Dessa forma, os motoristas profissionais de transporte de cargas podem estar mais expostos aos riscos cardiovasculares devido a características peculiares de sua profissão. Em geral, esses profissionais se alimentam em restaurantes, que, na sua maior parte, ofertam alimentos de alto valor calórico e baixo valor nutritivo, a associação de uma alimentação inadequada com o sedentarismo deixa-os mais expostos à obesidade e suas consequências (Girotto et al., 2020).

O objetivo desse estudo buscou analisar a produção científica acerca fatores que podem influenciar na promoção da saúde do caminhoneiro, estratificar o risco de doenças cardiovasculares, nos motoristas de caminhão, conhecer os fatores 
ocasionados por excesso de longas jornadas de trabalho, alcoolismos e DSTs.

\section{Metodologia}

O presente estudo trata-se de uma revisão de literatura referente aos fatores que podem influenciar na promoção da saúde do caminhoneiro. Para a construção desta revisão, foi operacionalizado um percurso metodológico por meio de seis etapas para o desenvolvimento: elaboração da pergunta norteadora, busca ou amostragem na literatura, coleta de dados, análise crítica dos estudos incluídos, discussão dos resultados e a apresentação da revisão integrativa (Macêdo \& Evargelandy, 2018).

Os estudos presentes no estudo foram selecionados e incluídos por meio dos seguintes critérios: artigos originais e completos disponíveis na íntegra na linguagem de inglês, espanhol e português, selecionados no período compreendido entre aos anos de 2016 a 2021. Utilizou-se como método de exclusão artigos duplicados, que não estavam disponíveis na integra de forma livre e gratuita, resumos e artigos da qual não correspondiam em seus objetivos os objetivos buscados nos resultados de pesquisa. A seleção dos estudos foi realizada de forma a ser revisada por um, pesquisador e o orientador da pesquisa.

Adotou-se para a formulação da pergunta norteadora a estratégia PICO conforme apresentada no Quadro 1, na qual o P (participantes ou problema): Caminhoneiros; o I (situação de interesse): Fatores prejudiciais; C (controle ou comparação): Comorbidades apresentadas; e por fim, $\mathrm{O}$ (desfecho): monitoramento de riscos.

Quadro 1. Estratégia PICO utilizada na pesquisa.

\begin{tabular}{|l|c|}
\hline P (participantes ou problema): & Caminhoneiros \\
\hline I (situação de interesse): & Fatores prejudiciais \\
\hline C (controle ou comparação): & Comorbidades apresentadas \\
\hline O (desfecho): & Monitoramento de riscos \\
\hline
\end{tabular}

Fonte: Autores (2021).

As buscas foram realizadas entre os meses de julho a agosto de 2021 pela as bibliotecas de saúde. Para o presente estudo, foram utilizados Descritores em Ciências da Saúde (DeCS): Promoção de saúde, qualidade de vida, autocuidado e transporte. Foram integrados com o operador lógico booleano "AND" nas bases de dados da MEDLINE, PUBMED e Scielo que mostrou uma busca mais precisa acerca do foco da temática, com isso foi possível encontrar os artigos para a elaboração dos resultados e discussão da pesquisa de forma rápida e precisa. 
Figura 1. Fluxograma da seleção independente dos pares dos estudos corpus da pesquisa de revisão integrativa da literatura. MEDLINE, PUBMED; SCIELO, 2021.

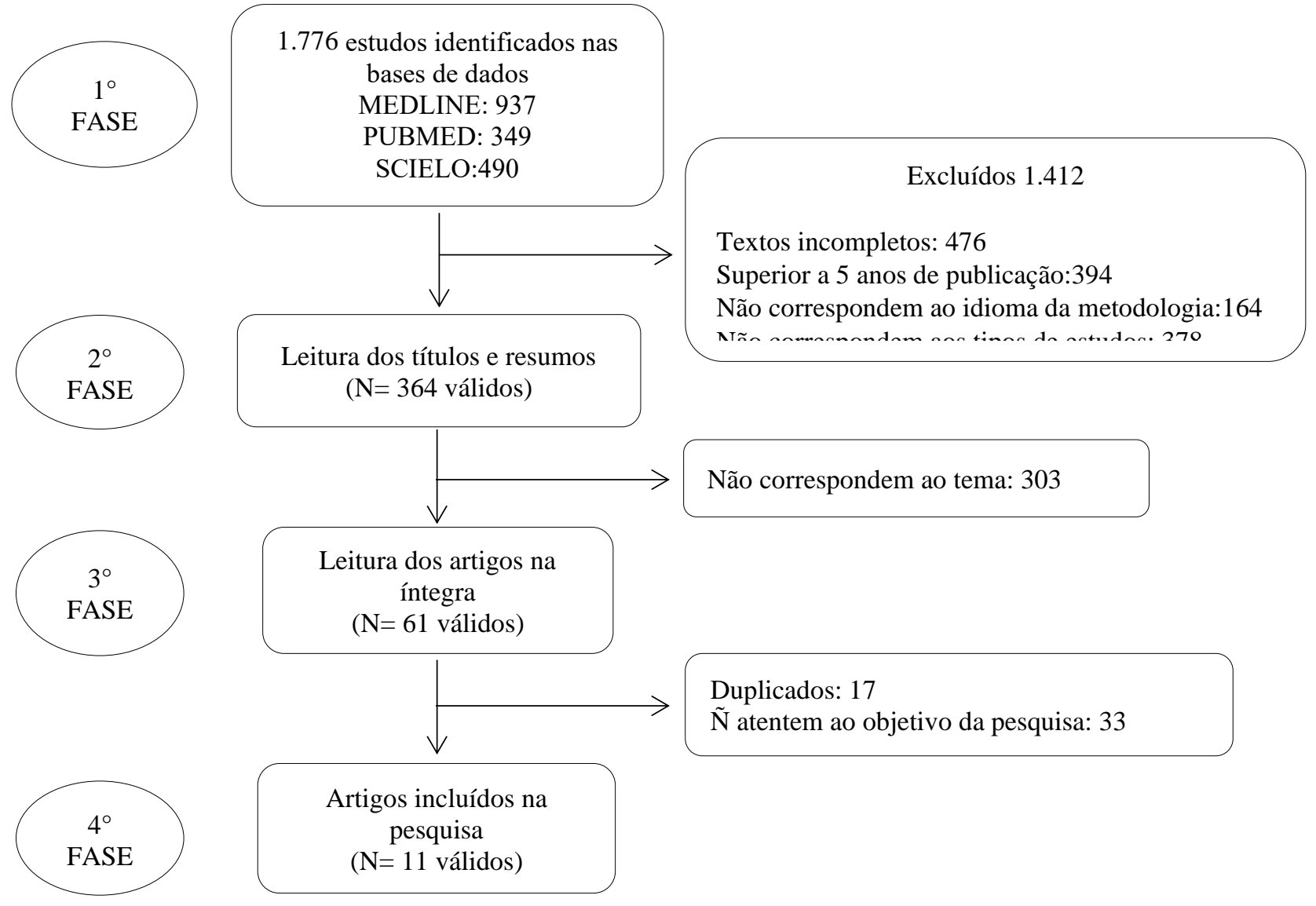

Fonte: Autores (2021).

\section{Resultados e Discussão}

Através da busca foram identificados 1.776 estudos, 937 na MEDLINE, 349 na PUBMED e 490 na SCIELO. Após a análise pela pesquisadora, 61 estudos foram selecionados para leitura. Desses estudos, 50 foram excluídos após a leitura, sendo selecionados 11 estudos validos para a composição da revisão.

A pesquisa foi realizada utilizando-se os Descritores em Ciências da Saúde (DeCS): Promoção de saúde, Qualidade de Vida, Autocuidado e Transporte, onde foram integrados com o operador lógico booleano “AND”. A síntese dos descritores utilizados, das bases de dados e das referências selecionadas está descrita na Tabela 1 abaixo: 
Tabela 1. Distribuição das referências obtidas nas bases de dados MEDLINE, PUBMED e SCIELO seguindo os descritores estabelecidos. Teresina, 2021.

\begin{tabular}{|c|c|c|c|}
\hline Base de dados & Descritores cruzados & $\begin{array}{c}\text { Referências } \\
\text { obtidas }\end{array}$ & Referências selecionadas \\
\hline MEDLINE & $\begin{array}{c}\text { Promoção de saúde } \\
\text { AND } \\
\text { Transporte }\end{array}$ & 564 & 3 \\
\hline MEDLINE & $\begin{array}{c}\text { Autocuidado } \\
\text { AND } \\
\text { Transporte } \\
\text { AND } \\
\text { Qualidade de Vida }\end{array}$ & 373 & 2 \\
\hline PUBMED & $\begin{array}{c}\text { Health Promotion } \\
\text { AND } \\
\text { Transportation }\end{array}$ & 278 & 2 \\
\hline PUBMED & $\begin{array}{c}\text { Self Care } \\
\text { AND } \\
\text { Transportation } \\
\text { AND } \\
\text { Quality of Life } \\
\end{array}$ & 71 & 1 \\
\hline SCIELO & $\begin{array}{c}\text { Promoção de saúde } \\
\text { AND } \\
\text { Transport }\end{array}$ & 234 & 1 \\
\hline SCIELO & $\begin{array}{c}\text { Autocuidado } \\
\text { AND } \\
\text { Transporte } \\
\text { AND } \\
\text { Qualidade de Vida }\end{array}$ & 256 & 2 \\
\hline TOTAL & & 1776 & 11 \\
\hline
\end{tabular}

Fonte. MEDLINE; PUBMED; SCIELO.

Após a análise das publicações selecionadas, fez-se o agrupamento dos estudos comparando os autores sobre os fatores que podem influenciar na promoção da saúde do caminhoneiro. O Quadro 2 exibe os estudos que compuseram esta revisão integrativa, no que se refere ao título, objetivo, abordagem, idioma e conclusão, todos referenciados de acordo com o artigo encontrado, especificando o ano de publicação e o periódico. 
Quadro 2. Categorização dos artigos encontrados referenciados pelos autores, objetivos, metodologia e a conclusão de cada estudo. $\mathrm{N}=15$. Teresina, 2021 .

\begin{tabular}{|c|c|c|c|c|}
\hline $\begin{array}{c}\text { AUTOR } \\
\text { (ANO) }\end{array}$ & TÍTULO & OBJETIVO & $\begin{array}{l}\text { MÉTODO } \\
\text { (LING.) }\end{array}$ & CONCLUSÃO \\
\hline $\begin{array}{l}\text { Morais; Borges } \\
\quad \text { (2017) }\end{array}$ & $\begin{array}{l}\text { Uma análise sobre } \\
\text { os riscos } \\
\text { ocupacionais dos } \\
\text { motoristas de } \\
\text { caminhão }\end{array}$ & $\begin{array}{l}\text { Revisar e analisar o que tem sido } \\
\text { publicado sobre o tema, bem } \\
\text { como identificar os fatores de } \\
\text { riscos ocupacionais a que estão } \\
\text { expostos estes trabalhadores, com } \\
\text { ênfase ao risco ergonômico. }\end{array}$ & $\begin{array}{l}\text { Revisão } \\
\text { teórico- } \\
\text { qualitativa, } \\
\text { (português) }\end{array}$ & $\begin{array}{l}\text { Foram elencadas diversas ideias de } \\
\text { autores referendos sobre os riscos e a } \\
\text { importância de um trabalho ergonômico } \\
\text { relacionando o social, o psicológico e o } \\
\text { físico dos trabalhadores em questão. }\end{array}$ \\
\hline $\begin{array}{l}\text { Rocha; Lemes; } \\
\text { Santos } \\
\text { (2017) }\end{array}$ & $\begin{array}{l}\text { Exposição de } \\
\text { caminhoneiros à } \\
\text { infecções } \\
\text { sexualmente } \\
\text { transmissíveis }\end{array}$ & $\begin{array}{l}\text { Analisar a exposição de } \\
\text { caminhoneiros à infecções } \\
\text { sexualmente transmissíveis. }\end{array}$ & $\begin{array}{l}\text { Descritivo e } \\
\text { exploratório } \\
\text { (português) }\end{array}$ & $\begin{array}{l}\text { A vulnerabilidade e o aumento da } \\
\text { incidência de IST caminham juntos; } \\
\text { sendo constatado entre os } \\
\text { caminhoneiros aspectos que os colocam } \\
\text { como vulneráveis: uso incorreto de } \\
\text { preservativos, afetado pelo uso de } \\
\text { álcool e outras drogas (ilícitas ou não), } \\
\text { assim como a busca inadequada ou } \\
\text { tardia para o tratamento. }\end{array}$ \\
\hline $\begin{array}{l}\text { Ribeiro; Nunes; } \\
\text { Oliveira (2017) }\end{array}$ & $\begin{array}{l}\text { Caracterização da } \\
\text { saúde dos } \\
\text { condutores de } \\
\text { veículos pesados e } \\
\text { perigosos que } \\
\text { trafegam em } \\
\text { rodovia federal no } \\
\text { estado de sergipe }\end{array}$ & $\begin{array}{c}\text { Caracterizar a saúde dos } \\
\text { condutores de veículos pesados e } \\
\text { perigosos que trafegam em } \\
\text { Rodovia Federal no Estado de } \\
\text { Sergipe, por meio de } \\
\text { levantamento realizado na ação } \\
\text { "Comando de Saúde nas } \\
\text { Rodovias". }\end{array}$ & $\begin{array}{l}\text { Quantitativa, } \\
\text { descritivo } \\
\text { com corte } \\
\text { transversal } \\
\text { (português) }\end{array}$ & $\begin{array}{l}\text { Encontramos em sua maioria indivíduos } \\
\text { sedentários, etilistas, tabagistas. } \\
\text { Associado a isso, encontramos risco } \\
\text { para o desenvolvimento de doenças } \\
\text { cardiovasculares elevado ao utilizarmos } \\
\text { dados antropométricos, avaliações de } \\
\text { exame físico e os critérios do Escore de } \\
\text { Framingham. }\end{array}$ \\
\hline $\begin{array}{l}\text { Alessi; Alves } \\
\text { (2016) }\end{array}$ & $\begin{array}{l}\text { Hábitos de vida e } \\
\text { condições de saúde } \\
\text { dos caminhoneiros } \\
\text { do Brasil: uma } \\
\text { revisão da literatura }\end{array}$ & $\begin{array}{l}\text { Revisar os hábitos de vida e } \\
\text { condições de saúde dos } \\
\text { caminhoneiros do Brasil. }\end{array}$ & $\begin{array}{l}\text { Revisão } \\
\text { qualitativa } \\
\text { (Português) }\end{array}$ & $\begin{array}{l}\text { Os estudos apontam que os } \\
\text { caminhoneiros possuem hábitos de vida } \\
\text { nocivos à saúde, tais como alimentação } \\
\text { não saudável, falta de atividade física, } \\
\text { tempo insuficiente de descanso, vícios } \\
\text { (cigarro e álcool), além do uso de } \\
\text { drogas, exposição a doenças } \\
\text { transmissíveis e da ausência de controle } \\
\text { periódico } \\
\text { em saúde. }\end{array}$ \\
\hline $\begin{array}{l}\text { Hino et al., } \\
\text { (2017) }\end{array}$ & $\begin{array}{l}\text { Análise dos } \\
\text { cuidados à saúde de } \\
\text { caminhoneiros }\end{array}$ & $\begin{array}{l}\text { Identificar variáveis acerca da } \\
\text { relação de caminhoneiros com os } \\
\text { serviços de saúde. }\end{array}$ & $\begin{array}{l}\text { Quantitativo, } \\
\text { transversal, } \\
\text { exploratório } \\
\text { (Português) }\end{array}$ & $\begin{array}{l}\text { O delineamento de estratégias para a } \\
\text { desmistificação de preconceitos } \\
\text { relacionados ao cuidado à saúde do } \\
\text { homem e a qualificação dos } \\
\text { profissionais de saúde para o } \\
\text { atendimento dessa população } \\
\text { contribuirão para a melhoria da } \\
\text { qualidade de vida e de saúde desses } \\
\text { profissionais. }\end{array}$ \\
\hline $\begin{array}{l}\text { Batista } \\
(2017)\end{array}$ & $\begin{array}{l}\text { Percepção sobre os } \\
\text { determinantes de } \\
\text { saúde em } \\
\text { caminhoneiros do } \\
\text { estado de sergipe }\end{array}$ & $\begin{array}{l}\text { Conhecer os determinantes de } \\
\text { saúde entre caminhoneiros do } \\
\text { estado de Sergipe, bem como a } \\
\text { percepção dos mesmos em } \\
\text { relação ao processo de saúde. }\end{array}$ & $\begin{array}{l}\text { Quali- } \\
\text { quantitativa } \\
\text { (Português) }\end{array}$ & $\begin{array}{l}\text { Os resultados sugerem perfil de } \\
\text { morbimortalidade para enfermidades } \\
\text { crônicas e relativa compreensão sobre } \\
\text { hábitos saudáveis, no entanto, com } \\
\text { dificuldades para colocar em prática, } \\
\text { alegando limitações devido às } \\
\text { condições de trabalho. }\end{array}$ \\
\hline $\begin{array}{l}\text { Gracindo; } \\
\text { Victorino; } \\
\text { Carvalho } \\
\text { (2019) }\end{array}$ & $\begin{array}{l}\text { Condição de saúde } \\
\text { de caminhoneiros } \\
\text { em rodovias do } \\
\text { paraná }\end{array}$ & $\begin{array}{l}\text { Analisar as condições de saúde e } \\
\text { fatores de risco para saúde dos } \\
\text { profissionais caminhoneiros. }\end{array}$ & $\begin{array}{l}\text { Transversal } \\
\text { descritivo } \\
\text { quantitativa } \\
\text { (Português) }\end{array}$ & $\begin{array}{l}\text { O presente estudo observou alta } \\
\text { prevalência de fatores de risco } \\
\text { modificáveis em profissionais } \\
\text { caminhoneiros quando comparados } \\
\text { com a população geral brasileira. }\end{array}$ \\
\hline $\begin{array}{l}\text { Oliveira; } \\
\text { Carlotto }\end{array}$ & $\begin{array}{l}\text { Fatores associados } \\
\text { aos transtornos }\end{array}$ & $\begin{array}{l}\text { Identificar os fatores de risco dos } \\
\text { Transtornos Mentais Comuns }\end{array}$ & $\begin{array}{l}\text { Quantitativo } \\
\text { descritivo }\end{array}$ & $\begin{array}{l}\text { Os resultados do estudo revelam um } \\
\text { perfil de risco constituído por variáveis }\end{array}$ \\
\hline
\end{tabular}




\begin{tabular}{|c|c|c|c|c|}
\hline (2020) & $\begin{array}{c}\text { mentais } \\
\text { comuns em } \\
\text { caminhoneiros }\end{array}$ & $\begin{array}{l}\text { (TMC) em uma amostra de } 565 \\
\text { caminhoneiros brasileiros. }\end{array}$ & (Português) & $\begin{array}{l}\text { laborais, psicossociais e estressores } \\
\text { ocupacionais, confirmando que fatores } \\
\text { ambientais e contextuais são } \\
\text { importantes para desenvolvimento dos } \\
\text { TMC. }\end{array}$ \\
\hline $\begin{array}{l}\text { Messias et al., } \\
\text { (2019) }\end{array}$ & $\begin{array}{l}\text { Being a truck driver } \\
\text { in Brazil: From } \\
\quad \text { implicit } \\
\text { self-stereotypes to } \\
\text { system justification }\end{array}$ & $\begin{array}{l}\text { Compreender as percepções que } \\
\text { os } \\
\text { caminhoneiros brasileiros têm de } \\
\text { si mesmos. }\end{array}$ & $\begin{array}{l}\text { Qualitativo } \\
\text { (Inglês) }\end{array}$ & $\begin{array}{l}\text { Este estudo pretendeu analisar } \\
\text { elementos significativos que pudessem } \\
\text { esclarecer os motivos de aceitação de } \\
\text { condições degradantes de trabalho entre } \\
\text { os membros deste grupo, que é } \\
\text { reconhecido como profundamente } \\
\text { desunido. }\end{array}$ \\
\hline $\begin{array}{l}\text { Magno } \\
(2019)\end{array}$ & $\begin{array}{l}\text { Estudos qualitativos } \\
\text { sobre } \\
\text { caminhoneiros e } \\
\text { HIV/aids: } \\
\text { contribuições para } \\
\text { análise de } \\
\text { vulnerabilidade }\end{array}$ & $\begin{array}{c}\text { Identificar e analisar criticamente, } \\
\text { com base no quadro analítico do } \\
\text { conceito de vulnerabilidade, } \\
\text { estudos qualitativos sobre a } \\
\text { vulnerabilidade de caminhoneiros } \\
\text { ao HIV. }\end{array}$ & $\begin{array}{l}\text { Qualitativo } \\
\text { (Português) }\end{array}$ & $\begin{array}{l}\text { Apesar das críticas apontadas, os } \\
\text { estudos qualitativos aqui revisados } \\
\text { trouxeram muitas contribuições ao } \\
\text { campo das investigações sobre } \\
\text { HIV/aids, principalmente por } \\
\text { aprofundar e resgatar questões } \\
\text { relevantes que outra metodologia não } \\
\text { abordaria. }\end{array}$ \\
\hline $\begin{array}{l}\text { Taube; Carlotto } \\
\text { (2018) }\end{array}$ & $\begin{array}{l}\text { Diferenças de } \\
\text { percepção de } \\
\text { estressores de } \\
\text { acordo com o tipo } \\
\text { de rota no trabalho } \\
\text { de caminhoneiros }\end{array}$ & $\begin{array}{l}\text { Verificar diferença na percepção } \\
\text { de estressores laborais em } \\
\text { caminhoneiros que realizam rotas } \\
\text { curtas e aqueles que percorrem } \\
\text { rotas longas no seu trabalho. }\end{array}$ & $\begin{array}{l}\text { Quali- } \\
\text { quantitativa } \\
\text { (Português) }\end{array}$ & $\begin{array}{c}\text { Identificou alguns estressores presentes } \\
\text { na atividade destes profissionais e as } \\
\text { diferenças nas rotas curtas e longas, } \\
\text { confirma a relevância de se considerar a } \\
\text { variável rota a partir de uma perspectiva } \\
\text { de organização do trabalho em } \\
\text { pesquisas que tenham como temática o } \\
\text { adoecimento ocupacional de } \\
\text { caminhoneiros. }\end{array}$ \\
\hline
\end{tabular}

Fonte: Autores (2021).

É notório de que os artigos oferecem uma riqueza de citações e informações de vários fatores que influenciam na promoção de saúde do caminhoneiro. Diante dos achados nas publicações o estudo permitiu fazer uma análise de algumas categorias temáticas que estão descritas abaixo:

\section{Fatores de risco a hipertensão e diabetes mellitus devido à má alimentação dos caminhoneiros}

No Brasil estudos identificaram inúmeros problemas para a saúde do caminhoneiro. As longas jornadas de trabalho com poucas interrupções fazem que o esse profissional acabe por negligenciar a sua saúde. Prazos e metas são estabelecidas por diversas empresas afim que a produtividade do trabalho seja aumentada. Com isso os caminhoneiros estão constante exposição de riscos físicos e ergonômicos. Além disso o desenvolvimento de certos hábitos contribui como fatores de risco para predisposição de várias doenças (Hino et al., 2017).

A má alimentação e hábitos, como o consumo de alimentos industrializados, fast-foods, a falta de exercício físico são uma das principais causas de aumento na prevalência dessas doenças crônicas (Ribeiro; Nunes; Oliveira, 2017). Por permanecerem um longo período fora de seus lares, acabam por realizar suas refeições em restaurantes de estadas, postos de gasolina ou até mesmo em uma cozinha improvisada fora do seu caminhão. Tais fatores acarretam em refeições precárias, com alimentos mal preparados e com alto teor calórico e valor nutritivo reduzido, ocasionando essas doenças crônicas e levando a esse trabalhador a obesidade (Alessi; Alves, 2016).

Ribeiro, Nunes e Oliveira (2017) relatam que, cerca de 49,8\% são etilistas, 38,1\% obesos, $63,2 \%$ sedentários, e possuem sonolência diurna excessiva por conta de suas jornadas de trabalho sendo de entre de 7 a 11 horas e todos possuem um alto e médio risco para doenças cardiovascular onde 60,8\% apresentaram alteração na circunferência abdominal e tiveram alteração no padrão visual. 
Por se tratar de um público que tem pouco disponibilidade de tempo, os caminhoneiros possuem uma certa dificuldade para realização de atividades preventivas de atenção primaria e secundaria. A procura desses profissionais aos serviços de saúde geralmente ocorre em funções de situações extremas. Esta busca está relacionada ao aparecer indícios do acometimento de alguma doença (Batista, 2017).

Esses problemas relacionados podem ser minimizados, se cada empregador buscar enquadrar em sua empresa, dentro dos padrões estabelecidos pelas normas. É necessário o aprimoramento de táticas e técnicas com o intuito de orientar sobre os riscos de saúde no qual esses profissionais estão expostos por conta de suas práticas irregulares. Quanto mais precocemente estas doenças forem detectadas, medidas farmacológicas e não farmacológicas podem ser implementadas. Tais intervenções têm como finalidade o controle dessas doenças (Morais; Borges, 2017).

\section{Excesso das longas jornadas de trabalho}

Em 2015 foi constituído a Lei $\mathrm{n}^{\circ}$ 13.103, estabelecendo que os caminhoneiros devem possuir 8h/dia de jornada de trabalho, respeitando um período de 11h/dia de descanso. Esta Lei como objetivo evitar acidentes nas rodovias e estradas do Brasil. O motorista não deve dirigir por mais de cinco horas interruptas. Esse tempo deve ser fracionado, pois a falta de atenção, cansaço e o sono, são um dos principais fatores de acidentes no transito. Estima-se que mais de $32 \%$ dos acidentes são ocasionados pela falta do descanso apropriado e cerca de 6\% desses acidentes são fatais (Gracindo; Victorino; Carvalho, 2019).

Infelizmente grande parte dos motoristas de transporte de cargas enfrentam uma longa jornada de trabalho irregular, onde permanece acordado muitas das vezes mais que 18h/dia. Estudos demonstram que esse tempo de irregular reduz o desempenho psicomotor. Esses trabalhadores de turno irregular são geralmente motoristas de caminhão autônomo ou não assalariado que perfaz jornadas superiores a 12/14 horas em horários irregulares e em turnos diversificados para atender a demanda e o também e os prazo de entrega da carga, obtendo 4/6 horas de sono por dia (Taube; Carlotto, 2018).

Por conta de sua necessidade de se manter em alerta ou acordado para que atinja os seus objetivos e metas, o profissional caminhoneiro acaba por utilizando meios para que seu sono seja inibido, recorrendo o uso de álcool e drogas ilícitas. Onde a utilização dessas substancias acabam inibindo suas atividades psicomotoras levando-os a sua dependência. Sendo também uma das principais causas de acidentes nas rodovias (Messias et al., 2019).

\section{Alcoolismo, drogas e rico a DSTs}

Estudos demonstram que cerca de 50\% dos motoristas de transportes de cargas já dirigiram logo após ter feito o consumo de álcool e 51\% após ter utilizado drogas. Alessi e Alves (2016), relatam que as drogas utilizadas mais comuns são a maconha, cocaína, antidepressivos, sedativos/ ansiolíticos, benzodiazepínicos e anfetamínicos. Além da utilização dessas substancia psicoativas, muitos destes caminhoneiros costumam dirigir em alta velocidade sobrepondo a que é permitido para a via.

Se sobressaindo o uso de anfetaminas os "rebites". Em estudos onde constatou-se que 66\% dos caminhoneiros tinham hábito do consumo, dos quais $27 \%$ faziam uso diário e $60 \%$ usavam de duas a três vezes por semana. Após o fim da meia-vida da droga, ocorre um efeito rebote, causando sonolência devido à anterior privação/diminuição do tempo de sono, provocando um mal desempenho na direção, sendo uma possível causa de acidentes de trânsito dentre essa classe de trabalhadores (Taube; Carlotto, 2018).

Acreditasse que o uso do álcool e outras drogas além de serem utilizadas para o seu aumento de produção, é utilizado por se sentir isolado e deprimido por conta da falta e da saudade da família, tornado essas substancias como um potencializador de sociabilidade, fazendo que crie laços denominados "amigos da estrada" (Oliveira; Carlotto, 2020). 
Essa solidão por conta do distanciamento familiar, faz com que o caminhoneiro busque formas de amenizar esse sentimento utilizando os serviços dos denominados "profissionais do sexo". Estas situações fazem com que fiquem ociosos facilitando as práticas de sexo e assédio de garotas (os) de programa.

Grande parte dos caminhoneiros relata que mantém relações sexuais com mais de uma pessoa. Uso incorreto de preservativos, desnorteados pelo consumo exacerbado álcool e das drogas, o baixo nível de conhecimento sobre hábitos saudáveis e modos de prevenir enfermidades, assim como a busca inadequada ou tardia para o tratamento, dando preferência a informações rápidas via colegas de trabalho ou balconistas de farmácias acaba que acarretando diversos problemas, como a gravidez indesejada e as doenças sexualmente transmissíveis (DSTs) (Rocha; Lemes; Santos, 2017).

Devido a maioria dos caminhoneiros serem casados ocasiona e uma serie conflitos nas relações com a sua parceria definida como principal (esposa), levando exposição às DSTs e entre outras repercussões. Esta é uma realidade que ocorre por todo o percurso de sua viagem, muitos caminhoneiros afirmam que possuem uma facilidade em obter parcerias sexuais, principalmente nos locais em que costumam fazer seu descanso.

Ao se contrair alguma infecção desse tipo o caminhoneiro é forçado a se afastar para buscar tratamento nos serviços de atendimento à saúde. A Postura frente a uma infecção por DSTs que não se tem cura, muitos mostraram certo despreparo emocional, pois temem a morte e segundo seria o confrontamento de seus familiares demonstrando sua conduta extraconjugal (Magno, 2019).

Oferecer compreensão da complexidade dos diversos fatores envolvidos no processo de saúde-doença-cuidado, faz com que se possa ter uma diminuição dos agravos a saúde. As ações para este público em saúde são raras e isoladas. Observa-se a necessidade de propostas e programas governamentais que visem a promoção e o cuidado com a saúde do caminhoneiro.

\section{Conclusão}

Observou-se em meio aos estudos que oferecer compreensão da complexidade acerca dos riscos e a exposição aos fatores que influenciam na saúde do caminhoneiro é de grande importância. Recomendasse para trabalhos produções literárias que abordem sobre os riscos que acometem o profissional por falta de conhecimento. A má alimentação, a falta de exercícios físicos, as longas jornadas de trabalho que lhes são impostas sem obedecer ao repouso recomendado junto a rotineira falta de proteção nas suas relações sexuais com os profissionais do sexo são um grande potencializador para adquirir doenças como, HAS e DM ou alguma DSTs, ocasionando severos possíveis problemas físicos e mentais. Posto isso, observa-se a necessidade de propostas e programas governamentais que visem a promoção e o cuidado com a saúde do caminhoneiro.

\section{Referências}

Alessi, A. \& Alves, M. K. (2016) Hábitos de vida e condições de saúde dos caminhoneiros do Brasil: uma revisão da literatura. Ciência \& Saúde, 8(3), 129-136.

Antoniassi Junior, G., Melo, H. C. S., Mendes, D. F., Silva, L. A. M. Oliveira, R. F. S., \& Gaya, C. M. (2016). O uso de drogas por motoristas caminhoneiros e o comportamento de risco nas estradas. Revista de Epidemiologia e Controle de Infecção, 6(4), 1-5.

Batista, A. M. F. (2017). Percepção sobre os determinantes de saúde em caminhoneiros do estado de sergipe: um estudo qualiquantitativo. 2017.118 f. Dissertação (Mestrado) - Curso de Ciências da Saúde., Universidade Federal de Sergipe-Ufse, Aracaju.

Fogaça, L. F., Santos, C.S., Souza, E. D., \& Morais, R. T. P. (2012). Identificação de caminhoneiros hipertensos da campanha de saúde na boleia de uma concessionária do interior de São Paulo. Nursing, São Paulo, 13(155), 204-208.

Francisco, P. M. S. B., Segri, N. J., Borim, F. S. A., \& Malta, D. C. (2018). Prevalência simultânea de hipertensão e diabetes em idosos brasileiros: desigualdades individuais e contextuais. Ciência \& Saúde Coletiva, 23(11), 3829-3840.

Girotto, E., Loch, M. R., Mesas, A. E., González, A. D., Guidoni, C. M., \& Andrade, S. M. (2020). Comportamentos alimentares de risco à saúde e fatores associados entre motoristas de caminhão. Ciência \& Saúde Coletiva, 25(3), 1011-1023.

Gracindo, C. V., Victorino, S. V. Z., \& Carvalho, M. D. B. (2019). Condição de saúde de caminhoneiros em rodovias do paraná. XI EPCC - Encontro Internacional de Produção Científica, 1(1), 1-8. 
Research, Society and Development, v. 10, n. 14, e482101422151, 2021

(CC BY 4.0) | ISSN 2525-3409 | DOI: http://dx.doi.org/10.33448/rsd-v10i14.22151

Hino, P., Francisco, T. R., Onofre, P. S. C., Santos, J. O., \& Takahashi, R. F. (2017). Análise dos cuidados à saúde de caminhoneiros. Rev Enferm Ufpe On Line, Recife, 11(11), 4741-4748.

Macêdo, C. S. \& Evangerlandy, G. M. (2018). Passo a Passo para Elaboração de Trabalhos Científicos. Teresina: F.C.s.M, 176.

Magno, L. (2019). Estudos qualitativos sobre caminhoneiros e HIV/aids: contribuições para análise de vulnerabilidade. Ciência \& Saúde Coletiva, 24(3), 715728.

Messias, J. C., Higuera, H. C. Silva, R. A., Facundo, G. N. S., \& Lessa, R. T. (2019). Being a truck driver in Brazil: from implicit self-stereotypes to system justification. Estudos de Psicologia, Campinas, 36(1), 1-11.

Morais, M. S. S., \& Borges, E. M. F. (2017). Uma análise sobre os riscos ocupacionais dos motoristas de caminhão. Revista Científica FacMais, 9(2), 199-225.

Notto, V. O., Brandão, V. L., Alves, A. F., Silva, L. M., \& D’Alessandro, W. B. (2017). Associação entre índice de massa corporal e circunferência da cintura com pressao arterial elevada em caminhoneiros. Revista cereus, 9(1), 163-177.

Oliveira, M. E. T. \& Carlotto, M. S. (2020). Factors associated with common mental disorders in truck drivers. Psicologia: Teoria e Pesquisa, 36(3653), 1-12.

Ramos, B. H., Almeida, M. F. C., Ribeiro, J. A. R., \& Silveira, C. A. (2018). Condições de vida, trabalho e saúde de motoristas de transporte de cargas. Rev Enferm Ufpe, Recife, 12(1), 150-159.

Ribeiro, C. L. S., Lima, D. M., \& Nunes, J. D. (2017). Caracterização da saúde dos condutores de veículo pesados e perigosos que trafegam em rodovia federal no estado de Sergipe. Caderno de Graduação - Ciências Biológicas e da Saúde - UNIT - Sergipe, 4(2), 35-52.

Rocha, E. M., Lemes, A. G., \& Santos, B. L. M. (2017). Exposição de caminhoneiros a infecções sexualmente transmissíveis. Journal Health Npeps, 2(1), 230240.

Rodrigues, L. F. S., Avelar, G. G., Toledo, J. O., Camargos, E. F., \& Nóbrega, O. T. (2018). Perfil de sono, variáveis clínicas e jornada de trabalho de caminhoneiros idosos e de meia-idade em rodovias. Geriatrics, Gerontology And Aging, 12(2), 96-101.

Silva, A. C., Rocha, Y. S., \& Lima, T. C. S. (2019). Saúde dos caminhoneiros e o reconhecimento social da profissão. Rev. Conexão Eletrônica, Três Lagoas, MS, 16(1), 181-189.

Taube, M. E. \& Carlotto, M. S. (2018). Diferenças de percepção de estressores de acordo com o tipo de rota no trabalho de caminhoneiros. Aletheia, 51(1-2), $52-67$. 